Check for updates

Cite this: Mater. Chem. Front., 2019, 3, 1613

Received 7th May 2019,

Accepted 3rd June 2019

DOI: 10.1039/c9qm00292h

rsc.li/frontiers-materials

\title{
Regulation of circular dichroism behavior and construction of tunable solid-state circularly polarized luminescence based on BINOL derivatives $\dagger$
}

\author{
Na Zhao, (D)*a Wangwang Gao, $\ddagger^{\mathrm{a}}$ Min Zhang, $\ddagger^{\mathrm{a}}$ Junfang Yang, ${ }^{\mathrm{b}}$ Xiaoyan Zheng, (D) *b \\ Yue Li, ${ }^{a}$ Rongrong Cui, ${ }^{a}$ Wei Yin ${ }^{a}$ and Nan Li (D)*a
}

\begin{abstract}
The development of chiral fluorescent materials has attracted great interest. $\left[1,1^{\prime}\right.$-binaphthalene]-2,2'diol (BINOL), a privileged $C_{2}$-symmetric molecular skeleton, is one of the most frequently used chiral optical backbones. However, a phenomenon of circular dichroism (CD) annihilation in the aggregated state was observed in BINOL based molecules, which limits the application of CD in the condensed state. In this work, by inserting various bridged alkyl chains into the hydroxyl groups of 3,3'-dicyanomethylene functionalized $(R)$-BINOL, a series of chiral luminogens (BINB- $n, n=1$ to 8 ) was obtained. All BINB-n luminogens exhibited aggregation-induced emission (AIE) characteristics and their molecular conformation was significantly dependent on the length of the alkyl chain, which further regulated their $C D$ behavior from annihilation to preservation in the aggregated state. Moreover, in virtue of the AIE characteristic of BINB-6, judiciously introducing suitable electron donors successfully realized enhanced and tunable solid-state circularly polarized luminescence with the maximum glum value of $1 \times 10^{-2}$.
\end{abstract}

\section{Introduction}

Chirality is a ubiquitous phenomenon in nature. Chiral molecules play crucial roles in most fundmental processes including the synthesis of deoxyribonucleic acid (DNA) or proteins, exploring new drug candidates and developing chiral functional materials. ${ }^{1}$ Unlike the significant progress of chiral molecules made in biological science and the pharmaceutical industry, their applications in the field of material science, especially in the fabrication of chiral organic materials, are relatively less investigated. Indeed, owing to their distinct optical information and angular independence, chiral organic materials are of pivotal importance for opto-electronic applications and chiral

\footnotetext{
${ }^{a}$ Key Laboratory of Macromolecular Science of Shaanxi Province, Key Laboratory of Applied Surface and Colloid Chemistry of Ministry of Education, Key Laboratory of the Ministry of Education for Medicinal Resources and Natural Pharmaceutical Chemistry, and School of Chemistry \& Chemical Engineering, Shaanxi Normal University, 710119 Xi'an, China.E-mail: nzhao@snnu.edu.cn,nli@snnu.edu.cn

${ }^{b}$ Beijing Key Laboratory of Photoelectronic/Electrophotonic Conversion Materials, Key Laboratory of Cluster Science of Ministry of Education, School of Chemistry and Chemical Engineering, Beijing Institute of Technology, 100081 Beijing, China. E-mail: xiaoyanzheng@bit.edu.cn

$\dagger$ Electronic supplementary information (ESI) available: Synthesis and characterization, optical spectra, crystal data and DFT calculations. CCDC 1823469-1823474 and 1856064. For ESI and crystallographic data in CIF or other electronic format see DOI: 10.1039/c9qm00292h

\$ These authors contributed equally to this work.
}

recognition processes. ${ }^{2-4}$ In order to obtain fine chiral information to clarify the working mechanism and further optimize the performance of chiral materials, various optical methods have been established. Among these methods, circular dichroism (CD) is without doubt the most commonly used technique. ${ }^{5} \mathrm{CD}$ spectrometry, which originated from ground-state electronic transition of $\pi$-conjugated chromophores, is extremely sensitive and accurate towards the chirality variations. Benefited by CD spectroscopy, the chirality information of chiral materials in different molecular states can be readily obtained.

$\left[1,1^{\prime}\right.$-Binaphthalene]-2,2'-diol (BINOL), one of the most privileged $\mathrm{C}_{2}$-symmetric molecules, was employed as an ideal chiral source to fabricate chiral organic materials. ${ }^{6}$ In recent years, an abnormal effect termed aggregation-annihilated CD (AACD) was reported in BINOL based molecules. Further studies revealed that the variation of the torsion angle $(\theta)$ between two naphthalene rings after molecular aggregation is responsible for this AACD behavior. ${ }^{7}$ The AACD phenomenon obstructed the application of $\mathrm{CD}$ in the aggregated state. Some elegant strategies were reported recently to surpass the annihilation of CD in BINOL derived molecules. ${ }^{7 a, 8}$ Nevertheless, precisely regulating the $\mathrm{CD}$ response in the process of aggregation through rational molecular design and synthesis is still a formidable challenge.

In addition, circularly polarized luminescence (CPL) is another key parameter of chiral materials which provides the excited-state chiral information. The CPL active materials, especially solid-state 
CPL materials have potential applications in the fields of 3D organic light-emitting diodes and optical data recording devices. ${ }^{9-14}$ Recently, a concept of aggregation-induced emission (AIE) has opened up great opportunities to develop solid-state CPL materials. ${ }^{15}$ For instance, naturally chiral auxiliaries such as amino acids, sugars, and lipids were decorated into achiral AIE luminogens (AIEgens) to obtain an enhanced CPL response in the condensed state. ${ }^{16}$ Meanwhile, axial BINOL was used as a chiral source to construct solid-state CPL materials. ${ }^{17}$ Beside superior chiral characteristics, BINOL also possess excellent luminescence properties due to its favorable $\pi$-conjugated structure and most of the reported studies focused on its CPL properties in the solution state. ${ }^{18}$ In contrast, development of enhanced and tunable solid-state CPL materials using BINOL as a chiral fluorescent skeleton is still rare.

Herein, by inserting various bridged alkyl chains into the hydroxyl groups of 3,3'-dicyanomethylene functionalized ( $R$ )-BINOL, a series of chiral luminogens (BINB-n, $n=1$ to 8) was obtained (Fig. 1). All BINB-n exhibited typical AIE features. Meanwhile, the molecular conformation of BINB- $\boldsymbol{n}$ was successfully controlled by the length of the alkyl chain, which resulted in the $\mathrm{CD}$ behavior converted from annihilation to preservation. Combining the experimental result with molecular dynamic simulation, the molecular conformation of BINB- $\boldsymbol{n}$ significantly influenced the change of $\theta$ during the aggregation process, and further affected their CD behaviors. Moreover, thanks to the AIE characteristic, introducing various electron donors into BINB-6 successfully yielded enhanced and tunable solid-state CPL.

\section{Results and discussion}

Following the molecular design concept, we began our investigation by introducing bridged alkyl chains with different lengths into the hydroxyl groups of 3,3'-dicyanomethylene functionalized $(R)$-BINOL to form a series of bridged structures termed as BINB- $\boldsymbol{n}(n=1$ to 8$)$. All luminogens displayed a higher absorption peak at approximately $280 \mathrm{~nm}$ in acetonitrile solution, which can be attributed to the $\pi-\pi^{*}$ transition. The longest absorption band at around $340 \mathrm{~nm}$ was observed, which can be ascribed to intramolecular charge transfer

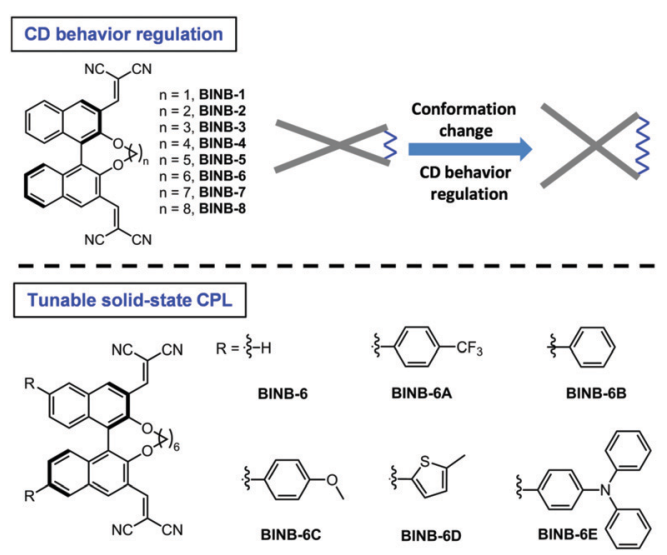

Fig. 1 Chemical structures of the (top) BINB- $\boldsymbol{n}$ and (bottom) BINB-6 derivatives.
(ICT) transition (Fig. S1A, ESI $\dagger$ ). Meanwhile, a remarkable solvent effect was observed for BINB-6 as an example, which further confirmed their ICT characteristics (Fig. S1D, ESI $\dagger$ ). ${ }^{19}$ After adding water into the acetonitrile solution, the emission intensity of all luminogens initially decreased owing to the intensive ICT process, but later increased and reached a maximum when the water fraction $\left(f_{\mathrm{w}}\right)$ was up to $99 \%$, demonstrating their typical AIE feature (Fig. 2A, B and Fig. S5, ESI $\dagger$ ). The AIE effect was mainly attributed to the restriction of the free motion of dicyanomethylene groups. ${ }^{7 b}$ It is noteworthy that the emission in the aggregated state exhibited a blue shift compared to that in the solution state, which could be caused by the reduction of polarity inside the aggregates. Molecular aggregation was further confirmed by dynamic light scattering measurement (Fig. S8, ESI $\dagger$ ).

Because of the identical absolute configuration of BINOL, similar Cotton effects were observed for BINB-1 to BINB-8 at 230, 270, 320 and $350 \mathrm{~nm}$ in their solution state (Fig. 2C and Fig. S11, ESI $\dagger$ ). The strong Cotton effects in the wavelength range from 230 to $320 \mathrm{~nm}$ can be attributed to the characteristic absorption of chiral binaphthyl moieties, while the CD peak at $350 \mathrm{~nm}$ originated from the whole molecular chirality. ${ }^{20}$ By increasing $f_{\mathrm{w}}$, the bridged chiral AIEgens presented distinct changes in CD response. Taking BINB-2 and BINB-6 as examples (Fig. 2C-E), almost no significant change in the CD spectra was detected when $f_{\mathrm{w}}$ was less than $60 \%$. However, with continuously increasing $f_{\mathrm{w}}$, the change of $\mathrm{CD}$ response exhibited a different tendency. For BINB-2, the CD signal dramatically decreased and approximately $25 \%$ intensity remained at $230 \mathrm{~nm}$ with the $f_{\mathrm{w}}$ up to 99\%. The decrease in CD response became weaker for BINB-6 while the CD intensity maintained more than $75 \%$ even at $f_{\mathrm{w}}$ of 99\%. Other BINB derivatives also displayed significantly alkyl
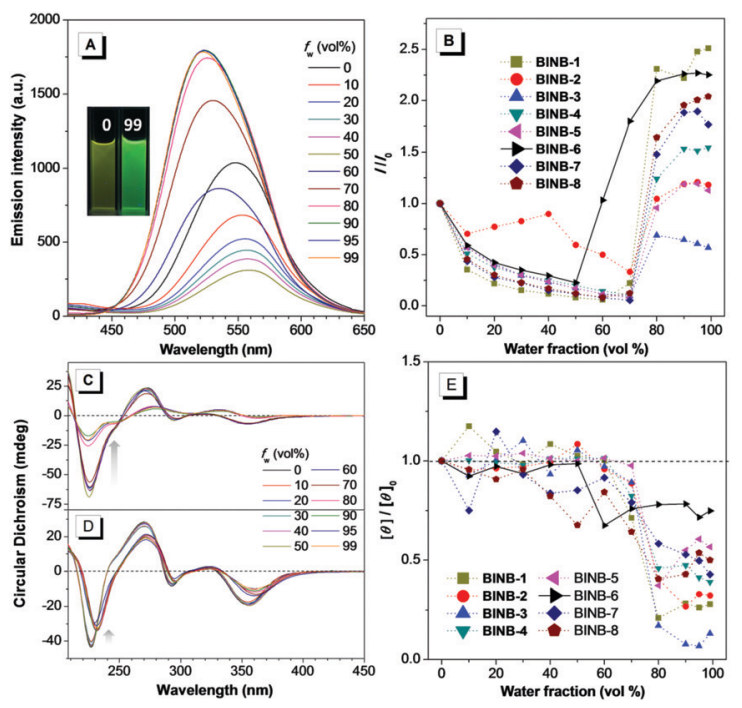

Fig. 2 (A) Emission spectra of BINB-6 in acetonitrile/water mixtures with different $f_{\mathrm{w}}$. (B) Plot of emission intensity versus the composition of $f_{\mathrm{w}}$ for BINB-1 to BINB-8. CD spectra of (C) BINB-2 and (D) BINB-6 in acetonitrile/water mixtures with different $f_{\mathrm{w}}$. (E) Plot of $C D$ signal intensity versus the composition of $f_{\mathrm{w}}$ for BINB-1 to BINB-8. Inset in (A): Photograph of BINB-6 in acetonitrile/water mixtures with 0 and $99 \% f_{\mathrm{w}}$ under $365 \mathrm{~nm}$ UV irradiation. 
chain dependent $\mathrm{CD}$ behavior with either preservation or annihilation. As described in Fig. 2E, BINB-6 displayed the utmost capability to preserve the $\mathrm{CD}$ intensity in the aggregated state. This remarkable conversion of CD behavior proved that the alkyl chain length can serve as an efficient way to modulate the CD property of chiral AIEgens in the process of molecular aggregation.

On the other hand, unbridged substituents with different steric hindrance, including methyl, tertbutyldimethylsilyl and tertbutyldiphenylsilyl groups, were introduced to generate BINUB-1 to BINUB-3 in order to investigate the influence of steric hindrance on the CD behavior (Scheme S3, ESI $\dagger$ ). A similar AIE phenomenon was observed for the three unbridged luminogens (Fig. S2A, B, S6 and S9, ESI $\dagger$ ). However, the CD signal gradually decreased with increasing $f_{\mathrm{w}}$, while the intensity at $230 \mathrm{~nm}$ dropped more than $55 \%$ at $f_{\mathrm{w}}$ of $99 \%$ (Fig. S2C, D and S12, ESI $\dagger$ ). This result suggested that, due to the flexible nature of the unbridged BINOL backbone, varying the degree of steric hinderance of the unbridged groups cannot effectively restrain the CD annihilation effect.

To determine the underlying reasons for the special optical behavior of these bridged chiral AIEgens, single crystals of the corresponding AIEgens were grown by slow evaporation from a suitable mixture of solvents. All single crystals of BINB- $\boldsymbol{n}$ were successfully obtained except BINB-3 and BINB-6 owing to their poor crystallization ability. Fortunately, a single crystal of the BINB-6 derivative that bears a trifluoromethylphenyl group at the $6,6^{\prime}$-position (BINB-6A), was obtained. ${ }^{21}$ The single crystal data are summarized in Tables S6 and S7 (ESI $\dagger$ ). As shown in Fig. 3 and Fig. S14-S20 (ESI $\dagger$ ), all corresponding molecules adopted highly nonplanar configurations inherited from BINOL while multiple intermolecular $\mathrm{C}-\mathrm{H} \cdots \mathrm{N}$ interactions $(2.472-2.645 \AA$ A) were found throughout most of the crystal lattice. The twisted structure combined with weak intermolecular interactions helped to rigidify the molecular conformation, prevented the molecules from forming $\pi-\pi$ or dipole-dipole interactions, and endowed BINB- $\boldsymbol{n}$ with high solid-state emission efficiency.
Further analysis of the molecular conformation revealed that the different lengths of alkyl chain at the 2,2'-position of BINB- $\boldsymbol{n}$ generated various $\theta$ values in the crystal. From BINB-1 to BINB-4, the $\theta$ value increased from 52.27 to $106.81^{\circ}$. By extending the alkyl chain from $n$-pentyl to $n$-heptyl (BINB-5 to BINB-7), the $\theta$ value showed little difference which was more than $111^{\circ}\left(111.52\right.$ to $\left.113.52^{\circ}\right)$. However, continuously prolonging the length of the alkyl chain (BINB-8), the $\theta$ value decreased to $89.83^{\circ}$. To understand the origin of alkyl chain-dependent CD behavior, molecular dynamics (MD) simulation of chiral AIEgens in a molecularly dissolved state was carried out by using BINB-2 and BINB-6A as representative examples (Fig. S21, ESI $\dagger$ ). The simulation results indicated that the $\theta$ value was $71.00^{\circ}$ and $111.00^{\circ}$ for BINB-2 and BINB-6A in their dissolved state. The change of $\theta$ value between the dissolved and crystal state was $9.68^{\circ}$ and $0.52^{\circ}$ for BINB-2 and BINB-6A, respectively. Clearly, the variation of the $\theta$ value for BINB-6A is smaller than that of BINB-2, which resulted in the preservation of the $\mathrm{CD}$ response of BINB-6A rather than BINB-2 in their aggregated state. $^{8,22}$

Since BINB-6 exhibited a relatively notable AIE effect, we expected to construct chiral AIEgens with tunable emission by introducing different donors into BINB-6 as suitable donor-acceptor systems can sufficiently control the separation of the highest occupied molecular orbital and lowest unoccupied molecular orbital. $^{19,23}$ The electron-donating potential increased in the following order: 4-(trifluoromethyl)phenyl, phenyl, 4-methoxyphenyl, 5-methylthiophenyl, and 4-(diphenylamino)phenyl, which formed a series of chiral AIEgens (BINB-6A to BINB-6E). The ICT absorption bands exhibited typical bathochromic-shift from 340 to $350 \mathrm{~nm}$, which is in line with the electron donating ability. Meanwhile, the longest CD response also shifted from 340 to $375 \mathrm{~nm}$ in the order from BINB-6 to BINB-6E. Importantly, the installed different electron donor has negligible influence on $\mathrm{CD}$ behavior since the $\mathrm{CD}$ intensity of BINB-6 derivatives is preserved in the process of aggregation. As shown in Fig. 4D and Fig. S13 (ESI $\dagger$ ), the CD signals at around $260 \mathrm{~nm}$ for BINB-6 derivatives maintained

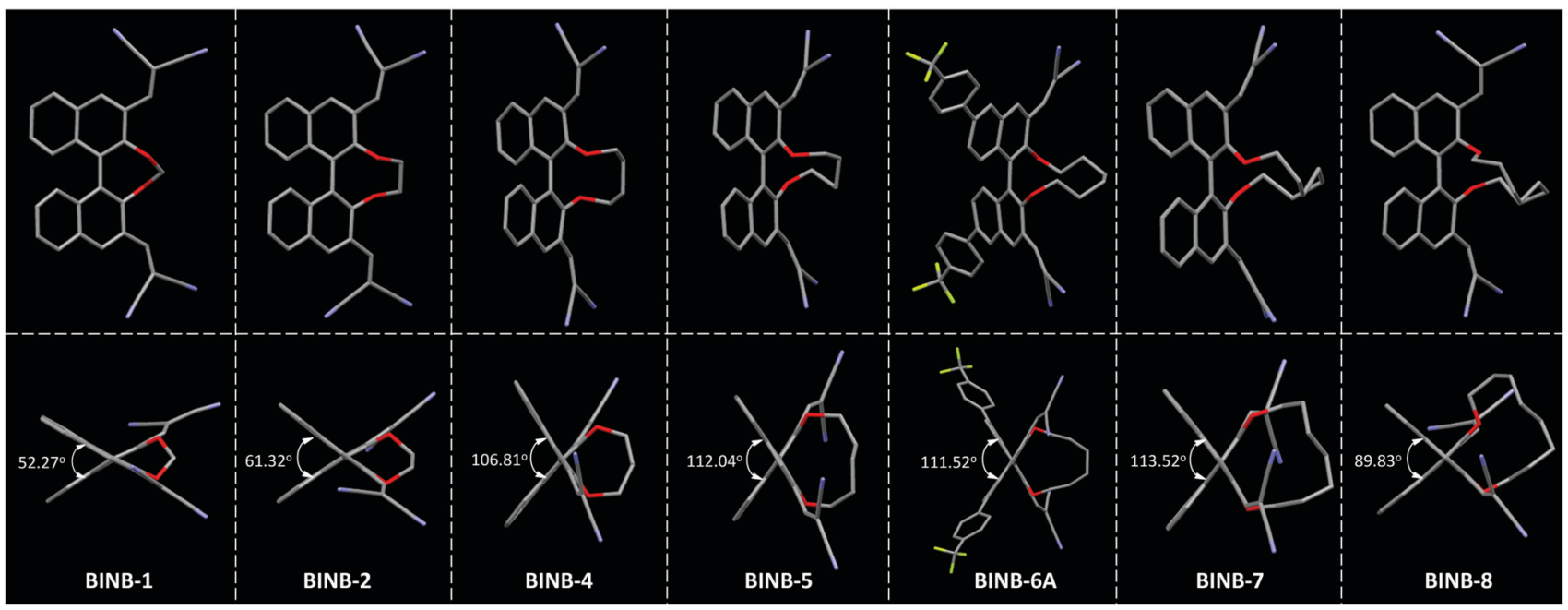

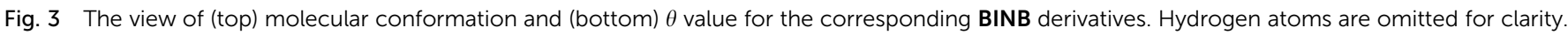



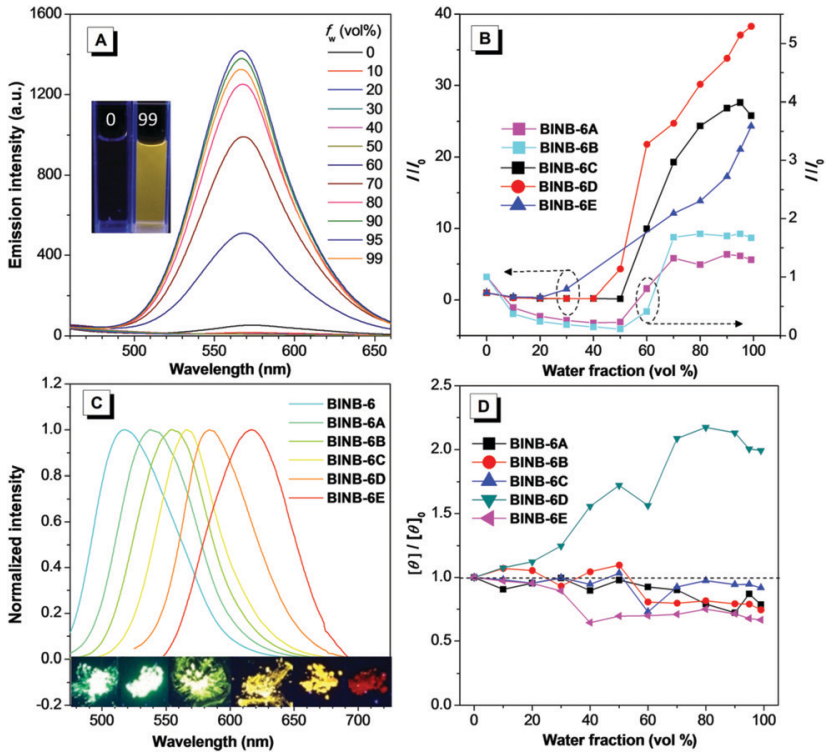

Fig. 4 (A) Emission spectra of BINB-6C in acetonitrile/water mixtures with different $f_{\mathrm{w}}$. (C) Emission spectra of BINB-6 to BINB-6E in their solid state. (B) Plot of emission intensity at specific wavelengths and (D) CD intensity at specific wavelengths versus the composition of $f_{\mathrm{w}}$ for BINB-6A to BINB-6E. Inset in (A): Photograph of BINB-6C in acetonitrile/water mixtures with 0 and $99 \% f_{\mathrm{w}}$ under $365 \mathrm{~nm}$ UV irradiation. Inset in (C): Photograph of BINB-6 to BINB-6E in solid state under $365 \mathrm{~nm}$ UV irradiation.

more than $75 \%$ at $f_{\mathrm{w}}$ of $99 \%$. It is interesting that 2 -fold enhancement of the CD intensity was observed for BINB-6D, which could be associated with its well-ordered aggregates. ${ }^{24}$

All BINB-6 derivatives exhibited enhanced emission in their aggregated state (Fig. 4A, B and Fig. S6, S9, ESI $\dagger$ ). Meanwhile, their solid-state quantum yields were higher than that in solution, which is consistent with their AIE feature (Table 1). Owing to the gradual strengthening of the electron-donating ability, the significant red-shift in solid-state emission ranging from 522 to $623 \mathrm{~nm}$ was observed (Fig. 4C). The CIE chromaticity diagrams (Fig. S3 and Table S1, ESI $\dagger$ ) demonstrated the same tunable emission color from green CIE $(0.2634,0.6195)$ to red CIE $(0.646,0.3536)$.

We further investigated the solid-state CPL properties of BINB-6 derivatives due to their AIE characteristics. Self-assembly of chiral organic molecules was reported to efficiently amplify the CPL signal

Table 1 Optical properties of BINB-6 derivatives

\begin{tabular}{|c|c|c|c|c|c|c|c|c|c|}
\hline \multirow[b]{2}{*}{ Compd. } & \multicolumn{4}{|c|}{ Solution state ${ }^{a}$} & \multicolumn{5}{|c|}{ Solid state } \\
\hline & $\begin{array}{l}\lambda_{\mathrm{abs}} \\
{[\mathrm{nm}]}\end{array}$ & $\begin{array}{l}\lambda_{\mathrm{em}} \\
{[\mathrm{nm}]}\end{array}$ & $\begin{array}{l}\Phi_{\mathrm{F}}^{b} \\
{[\%]}\end{array}$ & $\begin{array}{l}\tau_{\text {avg }}{ }^{c} \\
{[\mathrm{~ns}]}\end{array}$ & $\begin{array}{l}\lambda_{\mathrm{em}} \\
{[\mathrm{nm}]}\end{array}$ & $\begin{array}{l}\Phi_{\mathrm{F}}^{b} \\
{[\%]}\end{array}$ & $\begin{array}{l}\tau_{\text {avg }}{ }^{c} \\
{[\mathrm{~ns}]}\end{array}$ & $\begin{array}{l}\lambda_{\mathrm{CPL}}{ }^{d} \\
{[\mathrm{~nm}]}\end{array}$ & $\begin{array}{l}g_{\text {lum }} \\
\left(10^{-3}\right)\end{array}$ \\
\hline BIN & 338 & 547 & 8.6 & 498 & 518 & 11.2 & 5.38 & 5 & 0 . \\
\hline BINB-6A & 338 & 555 & 9.2 & 5.03 & 538 & 9.5 & 4.85 & 536 & -5.0 \\
\hline BINB-6B & 342 & 562 & 6.6 & 4.35 & 555 & 7.9 & 6.34 & 557 & 4 . \\
\hline BINB-6C & 345 & 575 & 0.5 & 0.99 & 566 & 6.8 & 7.54 & 580 & 1.0 \\
\hline BINB-6D & 338 & 577 & 0.7 & 3.59 & 584 & 4.0 & 6.15 & 597 & -10.0 \\
\hline BINB-6E & 350 & 623 & 0.3 & 4.35 & 617 & 0.7 & 9.06 & $-^{e}$ & $-^{e}$ \\
\hline
\end{tabular}

${ }^{a}$ Acetonitrile solution $(10 \mu \mathrm{M}) .{ }^{b}$ Absolute fluorescence quantum yield measured using the calibrated integrating sphere system. ${ }^{c}$ Mean fluorescence lifetime ( $\left.\tau_{\mathrm{avg}}\right)$ calculated using the equation $\tau_{\mathrm{avg}}=A_{1} \tau_{1}+$ $A_{2} \tau_{2}$. ${ }^{d}$ Self-assembled microstructure. ${ }^{e}$ No CPL was detected due to its microparticle form.

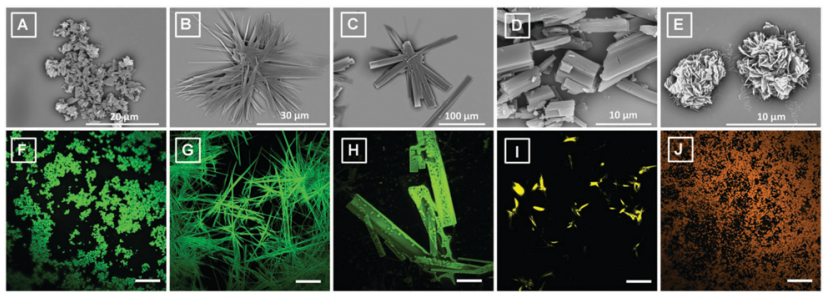

Fig. 5 The SEM images (A-E) and confocal fluorescent images $(F-J)$ of self-assembled BINB-6 to BINB-6D. Scale bar for F-J: $20 \mu \mathrm{m}$.

because of the chirality transfer from small molecules to the selfassembled network. ${ }^{15 d, 16 c, 25}$ Therefore, we prepared self-assembled samples of BINB-6 derivatives on a glass substrate by slowly evaporating the solvent (acetonitrile or dichloromethane). As shown in Fig. 5, the scanning electron microscope (SEM) images revealed that all BINB-6 derivatives successfully self-organized into microstructures with different shapes except for BINB-6E which formed microparticles. Detailed morphological analysis indicated that irregular tiny microslices and microneedles were formed for BINB-6 and BINB-6A, while BINB-6B and BINB-6C assembled into microrods. The microflowers constituted by secondary microplates were observed for BINB-6D. Under the confocal fluorescence microscope, all self-assembled microstructures displayed intense emission. At the same time, the emission color ranged from green to orange (520 to $580 \mathrm{~nm}$ ) following the order from self-assembled BINB-6 to BINB-6D (Fig. S4, ESI $\dagger$ ).

As expected, all self-assembled chiral AIEgens emitted CPL upon photoexcitation. As shown in Fig. 6A, the CPL peaks varied from 520 to $610 \mathrm{~nm}$ following the order from BINB-6 to BINB-6D, which cover the region from green to red. The magnitude of CPL was evaluated by the luminescence dissymmetry factor $\left(g_{\text {lum }}\right)$, which is defined as $g_{\text {lum }}=2 \times\left(I_{\mathrm{L}}-I_{\mathrm{R}}\right) /\left(I_{\mathrm{L}}+I_{\mathrm{R}}\right)$, where $I_{\mathrm{L}}$ and $I_{\mathrm{R}}$
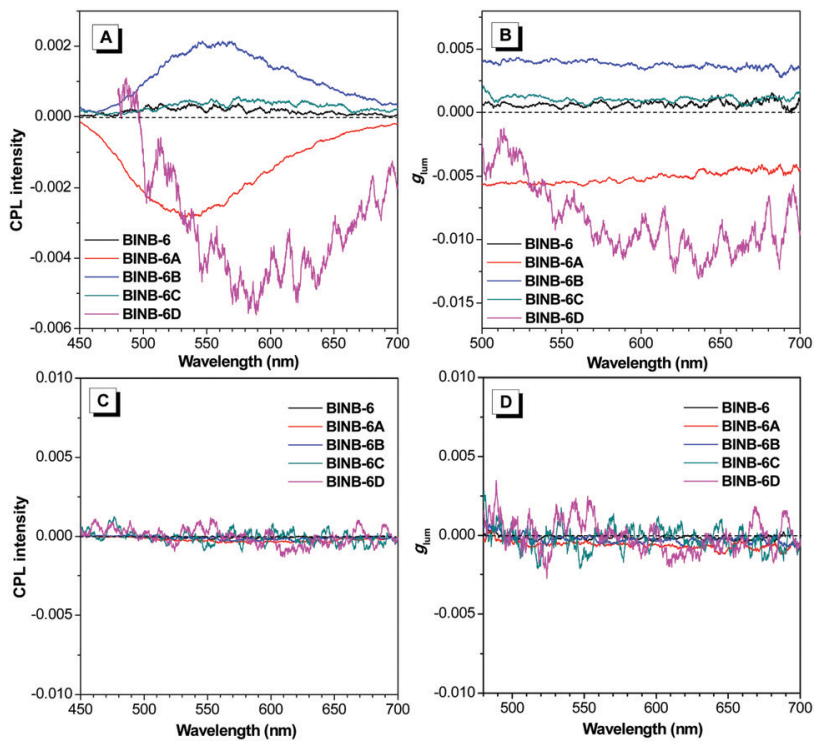

Fig. 6 The CPL spectra of (A) self-assembled microstructures and (C) the solution state of BINB-6 to BINB-6D. The corresponding $g_{\text {lum }}$ value of (B) self-assembled microstructures and (D) the solution state of BINB-6 to BINB-6D. 
refer to the intensity of left- and right-handed CPL, respectively. It is noted that the $g_{\text {lum }}$ factor of all self-assembled AIEgens was greater than $10^{-4}$, and the maximum $g_{\text {lum }}$ factor can reach up to $1 \times 10^{-2}$ for BINB-6D (Fig. 6B). In sharp contrast, almost no CPL response was detected in their dissolved state while nearly zero $g_{\text {lum }}$ was obtained (Fig. 6C and D). Intriguingly, BINB-6A and BINB-6D gave opposite CPL signals compared with those of BINB-6, BINB-6B and BINB-6C, which could be attributed to their different macroscopic chirality when they underwent a self-assembly process.

\section{Conclusions}

In conclusion, we developed a strategy to regulate $\mathrm{CD}$ behavior in the process of molecular aggregation through controlling the molecular conformation. By inserting different alkyl chains into the hydroxyl groups of BINOL derivatives, a series of chiral AIEgens (BINB-n) with different conformations was obtained. The conversion of the $\mathrm{CD}$ response from annihilation to preservation for BINOL derivatives was observed. Combining single crystal analysis and theoretical simulation, the different CD behavior was associated with their varied molecular conformation, which further influences the variation of $\theta$ value in the process of molecular aggregation. In addition, introducing diverse electron donors into the molecular backbone of BINB-6 yielded a series of chiral AIEgens with tunable emission. Moreover, these chiral AIEgens exhibited enhanced and tunable CPL after self-assembly.

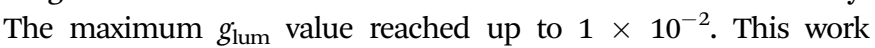
provides a general method to regulate the $\mathrm{CD}$ behavior for chiral luminogens as well as a facile approach to construct tunable solidstate CPL materials.

\section{Conflicts of interest}

There are no conflicts to declare.

\section{Acknowledgements}

We appreciate State Key Laboratory of Luminescent Materials and Devices for circularly polarized luminescence acquisition. This work was supported by the National Natural Science Foundation of China (21672135, 51403122 and 21402115), the Fundamental Research Funds for the Central Universities (GK201902006 and GK201702002), the Natural Science Foundation of Shaanxi Province (2018JM5086) and Funded Projects for the Academic Leaders and Academic Backbones, Shaanxi Normal University (18QNGG007).

\section{Notes and references}

1 (a) J. E. Hein and D. G. Blackmond, Acc. Chem. Res., 2012, 45, 2045-2054; (b) Z. Wang, W. Xu, L. Liu and T. F. Zhu, Nat. Chem., 2016, 8, 698-704; (c) W. H. Brooks, W. C. Guida and K. G. Daniel, Curr. Top. Med. Chem., 2011, 11, 760-770; (d) J. R. Brandt, F. Salerno and M. J. Fuchter, Nat. Rev.
Chem., 2017, 1, 0045; (e) S. R. LaPlante, L. D. Fader, K. R. Fandrick, D. R. Fandrick, O. Hucke, R. Kemper, S. P. F. Miller and P. J. Edwards, J. Med. Chem., 2011, 54, 7005-7022; $(f)$ H. Xu, S. Ding, W. An, H. Wu and W. Wang, J. Am. Chem. Soc., 2016, 138, 11489-11492.

2 (a) J. P. Riehl and F. S. Richardson, Chem. Rev., 1986, 86, 1-16; (b) G. Longhi, E. Castiglioni, J. Koshoubu, G. Mazzeo and S. Abbate, Chirality, 2016, 28, 696-707.

3 (a) M. Li, S. H. Li, D. D. Zhang, M. H. Cai, L. Duan, M. K. Fung and C. F. Chen, Angew. Chem., Int. Ed., 2018, 57, 2889-2893; (b) C. Liu, D. Yang, Q. Jin, L. Zhang and M. H. Liu, Adv. Mater., 2016, 28, 1644-1649.

4 (a) L. Pu, Acc. Chem. Res., 2017, 50, 1032-1040; (b) X. Zhang, J. Yin and J. Yoon, Chem. Rev., 2014, 114, 4918-4959; (c) L. Pu, Chem. Rev., 2004, 104, 1687-1716.

5 (a) C. Wolf and K. W. Bentley, Chem. Soc. Rev., 2013, 42, 5408-5424; (b) H. H. Jo, C. Y. Lin and E. V. Anslyn, Acc. Chem. Res., 2014, 47, 2212-2221; (c) G. Pescitelli, L. Di Bari and N. Berova, Chem. Soc. Rev., 2011, 40, 4603-4625; (d) N. Berova, L. Di Bari and G. Pescitelli, Chem. Soc. Rev., 2007, 36, 914-931.

6 J. M. Brunel, Chem. Rev., 2007, 107, PR1-PR45.

7 (a) H. K. Zhang, H. K. Li, J. Wang, J. Z. Sun, A. J. Qin and B. Z. Tang, J. Mater. Chem. C, 2015, 3, 5162-5166; (b) N. Li, H. L. Feng, Q. Gong, C. X. Wu, H. Zhou, Z. Y. Huang, J. Yang, X. H. Chen and N. Zhao, J. Mater. Chem. C, 2015, 3, 11458-11463.

8 H. K. Zhang, X. Zheng, R. T. K. Kwok, J. Wang, N. L. C. Leung, L. Shi, J. Z. Sun, Z. Y. Tang, J. W. Y. Lam, A. J. Qin and B. Z. Tang, Nat. Commun., 2018, 9, 4961.

9 (a) J. M. Han, S. Guo, H. Lu, S. J. Liu, Q. Zhao and W. Huang, Adv. Opt. Mater., 2018, 1800538; (b) H. Maeda, Y. Bando, K. Shimomura, I. Yamada, M. Naito, K. Nobusawa, H. Tsumatori and T. Kawai, J. Am. Chem. Soc., 2011, 133, 9266-9269.

10 (a) R. Carr, N. H. Evans and D. Parker, Chem. Soc. Rev., 2012, 41, 7673-7686; (b) C. T. Yeung, K. H. Yim, H. Y. Wong, R. Pal, W. S. Lo, S. C. Yan, M. Y. Wong, D. Yufit, D. E. Smiles, L. J. McCormick, S. J. Teat, D. K. Shuh, W. T. Wong and G. L. Law, Nat. Commun., 2017, 8, 1128; (c) R. Aoki, R. Toyoda, J. F. Kögel, R. Sakamoto, J. Kumar, Y. Kitagawa, K. Harano, T. Kawai and H. Nishihara, J. Am. Chem. Soc., 2017, 139, 16024-16027.

11 (a) R. A. van Delden, N. P. M. Huck, J. J. Piet, J. M. Warman, S. C. J. Meskers, H. P. J. M. Dekkers and B. L. Feringa, J. Am. Chem. Soc., 2003, 125, 15659-15665; (b) E. M. SánchezCarnerero, F. Moreno, B. L. Maroto, A. R. Agarrabeitia, M. J. Ortiz, B. G. Vo, G. Muller and S. de la Moya, J. Am. Chem. Soc., 2014, 136, 3346-3349; (c) K. Dhbaibi, L. Favereau, M. SrebroHooper, M. Jean, N. Vanthuyne, F. Zinna, B. Jamoussi, L. D. Bari, J. Autschbach and J. Crassous, Chem. Sci., 2018, 9, 735-742; (d) H. Nishimura, K. Tanaka, Y. Morisaki, Y. Chujo, A. Wakamiya and Y. Murata, J. Org. Chem., 2017, 82, 5242-5249; (e) S. Feuillastre, M. Pauton, L. H. Gao, A. Desmarchelier, A. J. Riives, D. Prim, D. Tondelier, B. Geffroy, G. Muller, G. Clavier and G. Pieters, J. Am. Chem. Soc., 2016, 138, 3990-3993. 
12 (a) S. H. Chen, D. Katsis, A. W. Schmid, J. C. Mastrangelo, T. Tsutsui and T. N. Blanton, Nature, 1999, 397, 506-508; (b) M. Oda, H. G. Nothofer, G. Lieser, U. Scherf, S. C. J. Meskers and D. Neher, Adv. Mater., 2000, 12, 362-365.

13 (a) F. D. Meng, Y. Z. Li, W. J. Zhang, S. H. Li, Y. W. Quan and Y. X. Cheng, Polym. Chem., 2017, 8, 1555-1561; (b) Y. Nagata, K. Takagi and M. Suginome, J. Am. Chem. Soc., 2014, 136, 9858-9861.

14 (a) D. Yang, P. F. Duan, L. Zhang and M. H. Liu, Nat. Commun., 2017, 8, 15727; (b) J. L. Han, J. You, X. G. Li, P. F. Duan and M. H. Liu, Adv. Mater., 2017, 29, 1606503; (c) F. Vera, R. Tejedor, M. P. Romero, J. Barberá, M. B. Ros, J. L. Serrano and T. Sierra, Angew. Chem., Int. Ed., 2007, 46, 1873-1877; (d) T. Goto, Y. Okazaki, M. Ueki, Y. Kuwahara, M. Takafuji, R. Oda and H. Ihara, Angew. Chem., Int. Ed., 2017, 56, 2989-2993.

15 (a) J. D. Luo, Z. L. Xie, J. W. Y. Lam, L. Cheng, H. Y. Chen, C. F. Qiu, H. S. Kwok, X. W. Zhan, Y. Q. Liu, D. B. Zhu and B. Z. Tang, Chem. Commun., 2001, 1740-1741; (b) J. Mei, N. L. C. Leung, R. T. K. Kwok, J. W. Y. Lam and B. Z. Tang, Chem. Rev., 2015, 115, 11718-11940; (c) J. Roose, B. Z. Tang and K. S. Wong, Small, 2016, 12, 6495-6512; (d) J. B. Xiong, H. T. Feng, J. P. Sun, W. Z. Xie, D. Yang, M. H. Liu and Y. S. Zheng, J. Am. Chem. Soc., 2016, 138, 11469-11472; (e) L. Shi, L. Y. Zhu, J. Guo, L. J. Zhang, Y. Shi, Y. Zhang, K. Hou, Y. L. Zheng, Y. F. Zhu, J. W. Lv, S. Q. Liu and Z. Y. Tang, Angew. Chem., Int. Ed., 2017, 56, 15397-15401.

16 (a) J. Z. Liu, H. M. Su, L. M. Meng, Y. H. Zhao, C. M. Deng, J. C. Y. Ng, P. Lu, M. Faisal, J. W. Y. Lam, X. H. Huang, H. K. Wu, K. S. Wong and B. Z. Tang, Chem. Sci., 2012, 3, 2737-2747; (b) Q. Ye, D. D. Zhu, H. X. Zhang, X. M. Lu and Q. H. Lu, J. Mater. Chem. C, 2015, 3, 6997-7003; (c) H. K. Li, J. Cheng, Y. H. Zhao, J. W. Y. Lam, K. S. Wong, H. K. Wu, B. S. Li and B. Z. Tang, Mater. Horiz., 2014, 1, 518521.
17 (a) S. W. Zhang, Y. X. Wang, F. D. Meng, C. H. Dai, Y. X. Cheng and C. J. Zhu, Chem. Commun., 2015, 51, 9014-9017; (b) J. Kumar, T. Nakashima, H. Tsumatori and T. Kawai, J. Phys. Chem. Lett., 2014, 5, 316-321; (c) Y. Sheng, D. Shen, W. J. Zhang, H. X. Zhang, C. J. Zhu and C. X. Cheng, Chem. - Eur. J., 2015, 21, 13196-13200; (d) F. Y. Song, Z. Xu, Q. S. Zhang, Z. Zhao, H. K. Zhang, W. J. Zhao, Z. J. Qiu, C. X. Qi, H. Zhang, H. H. Y. Sung, I. D. Williams, J. W. Y. Lam, Z. J. Zhao, A. J. Qin, D. G. Ma and B. Z. Tang, Adv. Funct. Mater., 2018, 28, 1800051.

18 (a) K. Takaishi, M. Yasui and T. Ema, J. Am. Chem. Soc., 2018, 140, 5334-5338; (b) E. M. Sánchez-Carnerero, A. R. Agarrabeitia, F. Moreno, B. L. Maroto, G. Muller, M. J. Ortiz and S. de la Moya, Chem. - Eur. J., 2015, 21, 13488-13500.

19 J. N. Zhang, H. Kang, N. Li, S. M. Zhou, H. M. Sun, S. W. Yin, N. Zhao and B. Z. Tang, Chem. Sci., 2017, 8, 577-582.

20 L. D. Bari, G. Pescitelli and P. Salvadori, J. Am. Chem. Soc., 1999, 121, 7998-8004.

21 CCDC 1823469 (BINB-1), 1823470 (BINB-2), 1823471 (BINB-4), 1823472 (BINB-5), 1823473 (BINB-6A), 1856064 (BINB-7) and 1823474 (BINB-8)†.

22 (a) S. F. Mason, R. H. Seal and D. R. Roberts, Tetrahedron, 1974, 30, 1671-1682; (b) T. Kimoto, N. Tajima, M. Fujiki and Y. Imai, Chem. - Asian J., 2012, 7, 2836-2841.

23 Z. Y. Zhang, B. Xu, J. H. Su, L. P. Shen, Y. S. Xie and H. Tian, Angew. Chem., Int. Ed., 2011, 50, 11654-11657.

24 E. Yashima, N. Ousaka, D. Taura, K. Shimomura, T. Ikai and K. Maeda, Chem. Rev., 2016, 116, 13752-13990.

25 (a) X. B. Shang, I. Song, H. Ohtsu, Y. H. Lee, T. M. Zhao, T. Kojima, J. H. Jung, M. Kawano and J. H. Oh, Adv. Mater., 2017, 29, 1605828; (b) G. Huang, R. Wen, Z. Wang, B. S. Li and B. Z. Tang, Mater. Chem. Front., 2018, 2, 1884-1892. 